\title{
Does steroid injection help patient rehabilitation after arthroscopic rotator cuff repair?
}

\author{
Sungwook Choi \\ Department of Orthopaedic Surgery, Jeju National University School of Medicine, Jeju, Korea
}

There are many factors to consider when treating rotator cuff tendinosis patients. Whether it is deciding between surgical repair or conservative treatment or determining the timing of a given method, such details should be discussed and chosen carefully. Once a surgical option is decided on, arthroscopic rotator cuff repair (RCR) is a standard option for improving postoperative pain and time to recovery compared to the open repair technique [1]. Studies show that successful healing leads to better strength and functional outcomes than incomplete healing [2]. Even though successful repair and healing of torn tendons influence patient outcome, a patient's preoperative status and postoperative rehabilitation are equally important. Although failed repair does not always correlate with low clinical results, as a surgeon, one should minimize any risk factors that may compromise the likelihood of successful treatment. Predisposing factors such as the patient's age, tear size, and tendon quality are unmodifiable, but we can optimize the rehabilitation process postoperatively. To improve the healing process for a repaired tendon, the patient is routinely immobilized for a certain period (for how long is another topic of discussion). This immobilization in turn can result in postoperative complications, which can act as sources of pain and stiffness [3]. Despite its possibility of side effects, postoperative subacromial steroid injections can reduce pain and stiffness [4].
In "Effects of steroid injection during rehabilitation after arthroscopic rotator cuff repair [5]," the authors compared the clinical outcomes of patients who received steroid injections with patients who did not. The pain and range of motion (ROM) recovery at 3-, 6-, and 24-month follow-up and functional score at 24-month follow-up were compared. In this study, a patient group who received ultrasound-guided subacromial steroid injection at 4 or 6 weeks after arthroscopic RCR exhibited reduced pain and ROM recovery time until three months after surgery, but there were no significant differences between the groups during further follow-up.

Adverse effects of steroid injections into the rotator cuff include infection, cartilage toxicity, and inhibiting normal inflammatory reactions that may hinder rotator cuff healing [6]. In vitro studies and retrospective studies demonstrated that corticosteroid exposure can result in increased failure of tendon healing, including poorer results with increased retears [7]. Compagnoni and Randelli [8] and Lubowitz et al. [9] also warned about the correlation between retears and infections associated with steroid injections perioperatively. In particular, the authors of "Effects of steroid injection during rehabilitation after arthroscopic rotator cuff repair [5]" chose the timing (4-6 weeks postoperatively) and type (ropivacaine) of injection with this in mind, in an effort to avoid normal inflammatory phases and cartilage toxicity. Similar

Received: August 9, $2021 \quad$ Accepted: August 12, 2021

Correspondence to: Sungwook Choi

Department of Orthopaedic Surgery, Jeju National University School of Medicine, 15 Aran 13-gil, Jeju 63241, Korea

Tel: +82-64-717-1690, E-mail: swchoi1115@gmail.com, ORCID: https://orcid.org/0000-0003-0319-6208

Financial support: This work was supported by the 2021 education, research and student guidance grant funded by Jeju National University. Conflict of interest: None.

Copyright@ 2021 Korean Shoulder and Elbow Society.

This is an Open Access article distributed under the terms of the Creative Commons Attribution Non-Commercial License (http://creativecommons.org/licenses/by-nc/4.0/) which permits unrestricted non-commercial use, distribution, and reproduction in any medium, provided the original work is properly cited. 
studies showed that perioperative injections can improve surgical results. Kim and Jung [10] showed that intraarticular steroid injection administered at 6 weeks postoperatively successfully reduced shoulder stiffness. Nakamura et al. [11] showed that preoperative hyaluronic acid injection improved functional outcomes.

Perioperative steroid injections for rotator cuff tear repairs is a highly debatable topic considering the possible side-effects. However, this does not mean steroid injection should be discarded altogether. Data regarding possible adverse effects can aid in properly weighing risk-benefit for RCR patients. As shown in the current study, short-term improvement in pain and ROM can greatly affect patient satisfaction and early recovery to daily life. Further studies considering prospective designs with more investigation into the optimal dosage, timing, and regimen of injection would be helpful.

\section{ORCID}

Sungwook Choi

https://orcid.org/0000-0003-0319-6208

\section{REFERENCES}

1. Severud EL, Ruotolo C, Abbott DD, Nottage WM. All-arthroscopic versus mini-open rotator cuff repair: a long-term retrospective outcome comparison. Arthroscopy 2003;19:2348.

2. Woolf SK. Editorial commentary: steroid injections prior to arthroscopic rotator cuff repair-is it time to rethink a conservative treatment paradigm. Arthroscopy 2019;35:714-6.

3. Parsons BO, Gruson KI, Chen DD, Harrison AK, Gladstone J,
Flatow EL. Does slower rehabilitation after arthroscopic rotator cuff repair lead to long-term stiffness. J Shoulder Elbow Surg 2010;19:1034-9.

4. Shin SJ, Do NH, Lee J, Ko YW. Efficacy of a subacromial corticosteroid injection for persistent pain after arthroscopic rotator cuff repair. Am J Sports Med 2016;44:2231-6.

5. Ha JW, Kim H, Kim SH. Effects of steroid injection during rehabilitation after arthroscopic rotator cuff repair. Clin Shoulder Elbow 2021;24:166-71.

6. Puzzitiello RN, Patel BH, Forlenza EM, Nwachukwu BU, Allen AA, Forsythe B, et al. Adverse impact of corticosteroids on rotator cuff tendon health and repair: a systematic review of basic science studies. Arthrosc Sports Med Rehabil 2020;2:e161-9.

7. Baverel L, Boutsiadis A, Reynolds RJ, Saffarini M, Barthélémy R, Barth J. Do corticosteroid injections compromise rotator cuff tendon healing after arthroscopic repair. JSES Open Access 2017;2:54-9.

8. Compagnoni R, Randelli P. Correlation between corticosteroid injections and surgical site infections in shoulder arthroscopy. Ann Transl Med 2020;8:513.

9. Lubowitz JH, Brand JC, Rossi MJ. Preoperative shoulder corticosteroid injection is associated with revision after primary rotator cuff repair. Arthroscopy 2019;35:693-4.

10. Kim IB, Jung DW. An intra-articular steroid injection at 6 weeks postoperatively for shoulder stiffness after arthroscopic rotator cuff repair does not affect repair integrity. Am J Sports Med 2018;46:2192-202.

11. Nakamura Y, Gotoh M, Mitsui Y, Nakamura H, Ohzono H, Okawa T, et al. Preoperative hyaluronic acid injection modulates postoperative functional outcome in patients undergoing arthroscopic rotator cuff repair. J Orthop Surg Res 2020;15:204. 